\title{
Countrywide implementation of whole genome sequencing: an opportunity to improve tuberculosis management, surveillance and contact tracing in low incidence countries
}

\section{To the Editor:}

In 2014, the World Health Organization (WHO) and European Respiratory Society published a framework for the elimination of tuberculosis (TB) in low incidence countries, describing the priority actions to achieve this goal. These include: investment on new tools for early diagnosis, access to universal drug susceptibility testing (DST), contact investigation and continuous surveillance [1]. A roadmap was recently proposed for Italy as model for low incidence countries [2], suggesting that TB elimination requires a concerted action fully coordinated at national and regional level. One of the major obstacles is the undetected transmission linked to international travels and migration [3]. Whole genome sequencing (WGS) with the cost of a conventional Mycobacterium tuberculosis DST assay supplies "all-in-one" information required to track transmissions with high resolution and to provide personalised management of $\mathrm{TB}$ cases in a timeframe shorter than the standard diagnostic pathway [4]. Here, we describe the results of a centralised WGS-based model for TB diagnosis and surveillance applied at regional level and how this model extended to a national scale in Italy or other low TB incidence countries could bring benefits to public health and patient management, saving costs for the health system.

In collaboration with the TB reference laboratory and clinical centres in Tuscany (RCM; Florence Careggi University Hospital and Pisa University Hospital), the Italian TB reference laboratory for molecular typing of mycobacteria and WHO supranational TB reference laboratory (SRL; Milan, Italy) performed WGS from early-positive BACTEC MGIT 960 (BD, Franklin Lakes, NJ, USA) cultures consecutively collected between February 2016 and September 2017 (20 months). Details on the study population are described in figure 1. Ethical clearance was not required as the routine workflow of the clinical laboratory and patient management were not modified. All data were anonymised. Primary cultures were heat inactivated at RCM and sent by courier to SRL, without any disruption of the diagnostic routine and not requiring specific transport biosafety measures. Testing at RCM included phenotypic DST for first-line and second-line drugs (in case of multidrug resistance) using MGIT, according to manufacturer's instructions and WHO recommendations [11]. Genomic DNA was extracted at SRL by automated Maxwell system (Promega, Madison, WI, USA) [12]. Illumina (San Diego, CA, USA) technology was used for paired-end WGS applying the Nextera XT DNA sample preparation kit and the "benchtop" MiniSeq platform (300 cycles). After quality check, reads were aligned to the H37Rv reference genome and variant calling was performed according to analysis pipelines calibrated in low burden settings and the most recent classification systems for resistance prediction, strain classification and phylogenetic reconstruction [5, 13, 14]. The pipeline was recalibrated to call drug resistance variants also at minor frequency.

WGS allowed generation of results within $72 \mathrm{~h}$ of delivery of the culture sample, while first-line DST results were obtained, on average, 28 days after culture positivity. A mean reads coverage of $73 \times$

@ERSpublications

Whole genome sequencing can be adopted at national level as routine assay for diagnosis, universal drug susceptibility testing and epidemiological investigation of TB cases in low incidence countries http://ow.ly/KA7I30jr0Wo

Cite this article as: Cabibbe AM, Trovato A, De Filippo MR, et al. Countrywide implementation of whole genome sequencing: an opportunity to improve tuberculosis management, surveillance and contact tracing in low incidence countries. Eur Respir J 2018; 51: 1800387 [https://doi.org/10.1183/13993003.00387-2018]. 




FIGURE 1 Countrywide implementation of whole genome sequencing (WGS): an opportunity to improve tuberculosis (TB) management, surveillance and contact tracing in low incidence countries. RCM: Tuscany regional reference centre for mycobacteria (Careggi University Hospital, Florence, Italy); MDR: multidrug-resistant; XDR: extensively drug-resistant; PPV: positive predictive value; NPV: negative predictive value; RIF: rifampicin; INH: isoniazid; PZA: pyrazinamide; ETB: ethambutol; cgMLST: (WGS-based) core genome Multilocus Sequence Typing; NA: information not available. \#: conferring-resistance mutations were classified according to [5-9]. ๆ: only isolates with both WGS and MGIT info available for each drug are reported in this analysis. For RIF, MDR: 284 samples, for INH, PZA, ETB: 285 samples. ${ }^{+}$: the 12 RIF-R strains are also MDR. \$: this analysis excludes cross-contaminations (total isolates considered: 274). Lineages: 1: East-African Indian (includes EAI and EAI Manila); 2: East-Asian (includes Beijing); 3: Indo-Oceanic (includes Delhi-CAS); 4: Euro-American (includes Cameroon, Haarlem, H37Rv-like, LAM, mainly T, S-type, Ural and X-type); 5: West Africa I; 6: West Africa II. SNP barcoding according to [10]. ${ }^{f}$ : standard epidemiological investigation conducted by the Unit of Hygiene and Public Health, Local Health Unit 10, in Florence. "\#: a cluster is defined by $\leqslant 6$ alleles difference. ${ }^{\text {} 17 . ~}$ repeated cultures at different times. ${ }^{++}$: for one patient, a third isolate was also available.

(range 24-286) was obtained from 298/328 positive MGIT cultures with WGS done (91\%). Negative predictive value (NPV) was $100 \%$ for the multidrug-resistant (MDR) phenotype and exceeded $96 \%$ for first-line drugs, showing that in a setting at low burden of resistance WGS predicts not only resistance but also susceptibility to first-line drugs, guiding treatment properly in the absence of phenotypic tests (figure 1). Pyrazinamide (PZA) was the only drug with reduced sensitivity (63\%): nine cases 
rifampicin-sensitive and phenotypically resistant to PZA did not show any mutation in PZA resistance associated genes. The unexpected high frequency of PZA resistance among non-MDR strains together with the well-known technical challenges of MGIT testing for this drug suggested that these cases could be interpreted as phenotypically false resistant [15]. 12 MDR isolates (from 11 patients) were correctly identified by WGS. One of them was extensively drug resistant due to phenotypic resistance to capreomycin not associated to variants in $r r s$ and $t l y A$ markers. Interestingly, 14 cases of resistance to fluoroquinolones (FQs) were promptly identified by WGS (from 11 patients) with 12 of them (10 patients) detected in non-MDR patients under FQ treatment for other infections. Overall, WGS showed $>96 \%$ diagnostic accuracy and NPV values for detection of resistance to first-line drugs compared to standard phenotypic testing, with extremely high performance for prediction of the MDR phenotype. WGS confirmed the heterogeneous TB epidemiology in Tuscany with the six major M. tuberculosis complex lineages (figure 1) causing disease in patients from over 30 countries: most represented sublineages were Haarlem, T, Beijing and LAM. Using a core genome multilocus sequence typing scheme (Ridom SeqSphere+ v4.1.9, threshold to define clusters: $\leqslant 6$ alleles) we identified 56 patients involved in transmission events with a clustering percentage of $12 \%$. Links were confirmed by single nucleotide variant (SNV)-based analysis (threshold to define clusters: $\leqslant 5$ SNVs) [13]. Cluster cases were immediately reported to RCM and local public health authorities that confirmed 52\% of links (figure 1). For the remaining 12 events, the standard contact tracing failed to retrieve epidemiological links: as expected, it is particularly cumbersome to track contacts among Italians and patients of mixed nationalities. Thus, WGS-based investigation represents an add-on value for identifying and interrupting transmission chains [6]. Mixed clusters involving Italian-born and foreigners were identified in 25\% (14/56) cases. Notably, WGS analysis rapidly reported seven cross-contamination events occurred in laboratories performing routine diagnosis, avoiding unnecessary treatment or further investigation.

We show here for the first time that the model generated in the UK is exportable and works in other low incidence European countries [12]. Indeed, WGS performed at the reference level could be the best intervention to perform systematic monitoring of drug resistance, to support individualised management of $\mathrm{TB}$ and to identify pockets of transmission in real time, overcoming the technical and budget limitations of the standard investigation. This strategy will ultimately limit the use of phenotypic DST to selected cases, thus saving costs. Recently, WALKER et al. [13] published a cross border cluster of MDR among Somali migrants: all patients reached Northern European countries after arriving to the Italian coasts. The two cases diagnosed in Italy were brought to public health attention after months of delay: WGS implementation at the national level would have contributed to their prompt treatment and reporting.

Italy notified 3769 incident cases in 2015 [16]: one to three equipped laboratories would be sufficient to handle sequencing of all cases with no delay. Italy is currently performing WGS only on MDR-TB cases to investigate dynamics of transmission, but in the absence of a central-regional coordination and commitment, the process is too slow and far from being comprehensive [17, 18]. In addition, to systematically understand the epidemiology and dynamic of transmission and to design appropriate interventions, typing should not be limited to resistant forms. As a further advantage, since second-line drugs are routinely tested after MDR is diagnosed, the information on second-line drugs provided by WGS could guide the prompt choice of appropriate regimens in case of need to deviate from first-line treatment, reducing empirical therapy.

As limitations, this approach provides high-quality results only when applied to cultured samples, although its application to sputum samples has been already described [4]. Moreover, the $9 \%$ WGS failure in this study is explained by the insufficient quantity of DNA obtained from 30 early positive cultures [19]. Samples were not resubmitted in that context. For its full implementation, the WGS technique will need to undergo validation procedures to meet clinical accreditation standards within the Italian regional system, and development of a comprehensive diagnostic report template to inform clinicians and the national health system. In addition, considering the need for batching samples in sequencing runs to limit costs, and for informatics infrastructure and high-level bioinformatics capacity, a centralised approach with links between the routine setting and the specialised laboratory performing the sequencing, would enable the efficient adoption of WGS as a routine assay for diagnosis, universal DST and epidemiological investigation of TB cases.

Andrea Maurizio Cabibbe $\oplus^{1}$, Alberto Trovato ${ }^{1}$, Maria Rosaria De Filippo ${ }^{1}$, Arash Ghodousi ${ }^{1}$, Laura Rindi ${ }^{2}$, Carlo Garzelli ${ }^{2}$, Simonetta Baretti ${ }^{3}$, Guendalina Allodi ${ }^{3}$, Roberta Mannino ${ }^{4}$, Gian Maria Rossolini ${ }^{4,5}$, Alessandro Bartoloni ${ }^{5,6}$, Enrico Tortoli ${ }^{1}$ and Daniela Maria Cirillo ${ }^{1}$

${ }^{1}$ Emerging Bacterial Pathogens Unit, IRCCS San Raffaele Scientific Institute, Milan, Italy. ${ }^{2}$ University of Pisa, Dept of Translational Research and New Technologies in Medicine and Surgery, Pisa, Italy. ${ }^{3}$ Unit of Hygiene and Public Health, 
Local Health Unit 10, Florence, Italy. ${ }^{4}$ Careggi University Hospital, Clinical Microbiology and Virology Unit, Florence, Italy. ${ }^{5}$ Dept of Experimental and Clinical Medicine, University of Florence, Florence, Italy. ${ }^{6}$ Careggi University Hospital, Infectious and Tropical Diseases Unit, Florence, Italy.

Correspondence: Daniela Maria Cirillo, Emerging Bacterial Pathogens Unit, Division of Immunology, Transplantation and Infectious Diseases, IRCCS Ospedale San Raffaele, Via Olgettina 58, 20132 Milan, Italy. E-mail: cirillo.daniela@hsr.it

Received: Feb 232018 | Accepted after revision: April 022018

Conflict of interest: None declared.

Support statement: This study was supported by the 2nd ERANet-LAC Transnational Joint Call on Research and Innovation (grant: TRANS-TB-TRANS PER-2012-ELAC2015/T08-0664). Funding information for this article has been deposited with the Crossref Funder Registry.

Sequences are available as fastq files in the Sequence Read Archive of the National Center for Biotechnology Information (accession number SRP135980), after removal of human reads.

\section{References}

1 Lönnroth K, Migliori GB, Abubakar I, et al. Towards tuberculosis elimination: an action framework for low-incidence countries. Eur Respir J 2015; 45: 928-952.

2 Blasi F, Matteelli A, Sotgiu G, et al. Moving towards tuberculosis elimination: a call for action from Italy and a possible model for other low tuberculosis incidence countries. Eur Respir J 2017; 49: 1602242.

3 Marais BJ, Walker TM, Cirillo DM, et al. Aiming for zero tuberculosis transmission in low-burden countries. Lancet Respir Med 2017; 5: 846-848.

4 Votintseva AA, Bradley P, Pankhurst L, et al. Same-day diagnostic and surveillance data for tuberculosis via whole-genome sequencing of direct respiratory samples. J Clin Microbiol 2017; 55: 1285-1298.

5 Feuerriegel S, Schleusener V, Beckert P, et al. PhyResSE: a web tool delineating Mycobacterium tuberculosis antibiotic resistance and lineage from whole-genome sequencing data. J Clin Microbiol 2015; 53: 1908-1914.

6 Arnold A, Witney AA, Vergnano S, et al. XDR-TB transmission in London: case management and contact tracing investigation assisted by early whole genome sequencing. J Infect 2016; 73: 210-218.

7 Farhat MR, Sultana R, Iartchouk O, et al. Genetic determinants of drug resistance in Mycobacterium tuberculosis and their diagnostic value. Am J Respir Crit Care Med 2016; 194: 621-630.

8 Walker TM, Kohl TA, Omar SV, et al. Whole-genome sequencing for prediction of Mycobacterium tuberculosis drug susceptibility and resistance: a retrospective cohort study. Lancet Infect Dis 2015; 15: 1193-1202.

9 Zignol M, Cabibbe AM, Dean AS, et al. Genetic sequencing for surveillance of drug resistance in tuberculosis in highly endemic countries: a multi-country population-based study. Lancet Infect Dis 2018; in press [https://doi. org/10.1016/S1473-3099(18)30073-2].

10 Coll F, McNerney R, Guerra-Assunção JA, et al. A robust SNP barcode for typing Mycobacterium tuberculosis complex strains. Nat Commun 2014; 5: 4812.

11 World Health Organization. Updated Interim Critical Concentrations for First-line and Second-line DST. www. stoptb.org/wg/gli/assets/documents/Updated\%20critical\%20concentration\%20table_1st\%20and\%202nd\%20line\% 20drugs.pdf Last updated: May 2012.

12 Pankhurst LJ, Del Ojo Elias C, Votintseva AA, et al. Rapid, comprehensive, and affordable mycobacterial diagnosis with whole-genome sequencing: a prospective study. Lancet Respir Med 2016; 4: 49-58.

13 Walker TM, Merker M, Knoblauch AM, et al. A cluster of multidrug-resistant Mycobacterium tuberculosis among patients arriving in Europe from the Horn of Africa: a molecular epidemiological study. Lancet Infect Dis 2018; 18: 431-440.

14 Miotto P, Tessema B, Tagliani E, et al. A standardised method for interpreting the association between mutations and phenotypic drug resistance in Mycobacterium tuberculosis. Eur Respir J 2017; 50: 1701354.

15 Miotto P, Cabibbe AM, Feuerriegel S, et al. Mycobacterium tuberculosis pyrazinamide resistance determinants: a multicenter study. MBio 2014; 5: e01819-e01814.

16 European Centre for Disease Prevention and Control. Tuberculosis Surveillance and Monitoring in Europe 2017. Stockholm, European Centre for Disease Prevention and Control/WHO Regional Office for Europe, 2017.

17 Fattorini L, Mustazzolu A, Borroni E, et al. Tuberculosis in migrants from 106 countries to Italy, 2008-2014. Eur Respir J 2016; 47: 1273-1276.

18 De Beer JL, Kodmon C, van der Werf MJ, et al. Molecular surveillance of multi- and extensively drug-resistant tuberculosis transmission in the European Union from 2003 to 2011. Euro Surveill 2014; 19: 20742.

19 Shea J, Halse TA, Lapierre P, et al. Comprehensive whole-genome sequencing and reporting of drug resistance profiles on clinical cases of Mycobacterium tuberculosis in New York State. J Clin Microbiol 2017; 55: 1871-1882. 